This item was submitted to Loughborough's Research Repository by the author.

Items in Figshare are protected by copyright, with all rights reserved, unless otherwise indicated.

\title{
Information and capital asset pricing
}

PLEASE CITE THE PUBLISHED VERSION

http://dx.doi.org/10.1080/1351847X.2010.495476

PUBLISHER

(c) Taylor and Francis

VERSION

AM (Accepted Manuscript)

LICENCE

CC BY-NC-ND 4.0

REPOSITORY RECORD

Li, Baibing, and Xiangkang Yin. 2019. "Information and Capital Asset Pricing". figshare. https://hdl.handle.net/2134/9160. 
This item was submitted to Loughborough's Institutional Repository (https://dspace.lboro.ac.uk/) by the author and is made available under the following Creative Commons Licence conditions.

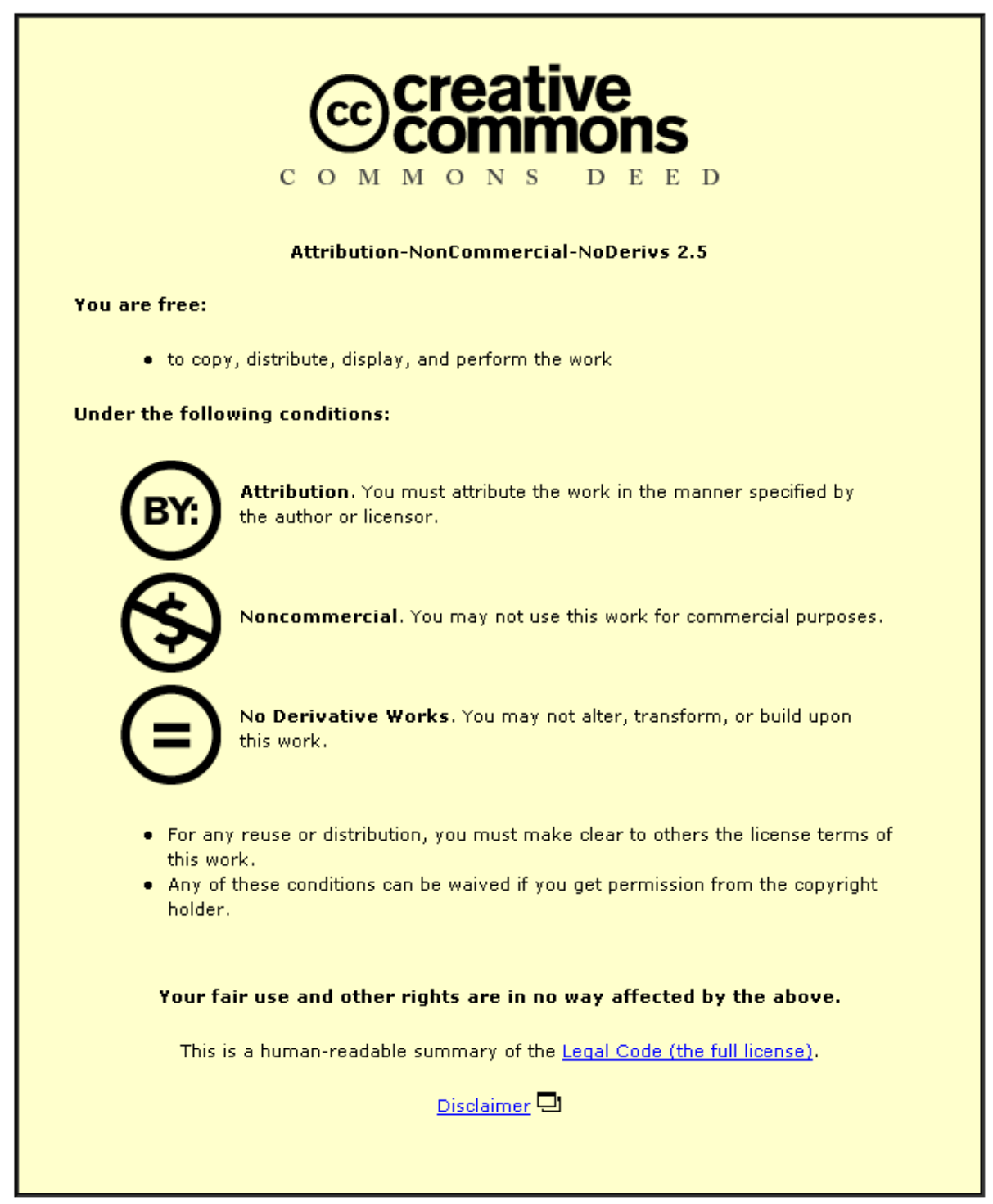

For the full text of this licence, please go to: http://creativecommons.org/licenses/by-nc-nd/2.5/ 


\title{
INFORMATION AND CAPITAL ASSET PRICING*
}

\author{
BAIBING LI \\ Business School \\ Loughborough University
}

\author{
XIANGKANG YIN \\ Corresponding author \\ School of Economics and Finance \\ La Trobe University \\ Bundoora, Victoria 3083 \\ Australia \\ Tel: 61-3-9479 2312 \\ Fax: 61-3-9479 1654 \\ Email: x.yin@latrobe.edu.au
}

\footnotetext{
* Some of theoretical results in this paper have been presented in an earlier working paper "Capital Asset Pricing with Asymmetric Information”. We are grateful to Buly Cardak, David Feldman, Peter Swan, Jianxin Wang, seminar participants at the University of New South Wales, the University of Sydney, the University of Nottingham, Loughborough University, La Trobe University, and participants in the $62^{\text {nd }}$ European Meeting of Econometrics Society.
} 


\title{
INFORMATION AND CAPITAL ASSET PRICING
}

\begin{abstract}
Investors in a market frequently update their diverse perceptions of the values of risky assets, thus invalidating the classic CAPM's assumption of complete agreement among investors. To accommodate information asymmetry and belief updating, we have developed an empirically testable Information-adjusted CAPM, which states that the expected excess return of a risky asset/portfolio is solely determined by information-adjusted beta rather than market beta. The model is then used to analyze empirical anomalies of the classic CAPM, including a flatter relation between average return and market beta than the CAPM predicts, a non-zero Jensen's alpha, insignificant explanatory power of market beta, and size effect.
\end{abstract}




\section{Introduction}

The Capital Asset Pricing Model (CAPM) is one of the corner stones of modern finance theory. The Sharpe-Lintner version of the CAPM (Sharpe (1964) and Lintner (1965)) postulates that the expected return on a risky asset or a portfolio of assets is a linear function of its market beta, with a positive slope equal to the expected excess return on the market portfolio and an intercept equal to the riskless interest rate. It implies that market beta is the only variable which can and is required to explain the systematic risk of securities. However, such an insightful and elegant theory has met great challenges in empirical tests. ${ }^{1}$ Empirical evidence shows that the slope in a regression of return on market beta is small, resulting in a much flatter regression line than the theoretical security market line. The intercept of such regressions systematically deviates from the riskless rate. Many studies also report that market beta does not have power in explaining return variation. Other variables, including the size of a security, play a substantial role in explaining return variation and have even greater explanatory power than market beta.

Like other theories, the Sharpe-Lintner CAPM is built on a series of assumptions simplifying the complexity of the real world. Among them, two assumptions are relatively restrictive.

"The first assumption is complete agreement: given market clearing asset prices at $t-1$, investors agree on the joint distribution of asset returns from $t$ -1 to $t$. And this distribution is the true one-that is, it is the distribution from which the returns we use to test the model are drawn. The second assumption is that there is borrowing and lending at a risk-free rate, which is the same for all investors and does not depend on the amount borrowed or lent.” (Fama and French (2004), p. 26. Italic is original.)

Black (1972) replaces the second assumption by assuming no risk-free asset and no risk-free borrowing or lending. The Black version of the CAPM is consistent with a

\footnotetext{
${ }^{1}$ For recent surveys on the theory and empirical evidence of the CAPM, see Fama and French (2004).
} 
flatter slope between expected return and market beta and a non-zero Jensen's alpha but it still cannot explain other anomalies reported in empirical studies. ${ }^{2}$

This paper intends to relax the complete agreement assumption and develop a new asset pricing model with improved predictions. Hence, it is assumed that no investor in a financial market knows the true distribution of asset returns. But they receive signals revealing the information of the true values of risky assets. Investors incorporate the new information with their prior beliefs to refine their knowledge of the market and security returns. Because investors may receive asymmetric information, their posterior beliefs about the return distribution are heterogeneous.

When the assumption of complete agreement is relaxed, the information received by investors or more precisely the distribution of information index is crucial. The information index of a risky asset depicts the effective information possessed by investors. ${ }^{3}$ If the information index takes the same value across all securities because of certain very particular market conditions, the market beta captures the systematic risk of an asset, in turn, the Sharpe-Lintner CAPM holds. However, the information index generally varies across securities so that the systematic risk of an asset cannot be properly measured by the market beta. Hence, we need to introduce a new benchmark portfolio - the Information-adjusted Market Portfolio (IaMP). The weight on each risky asset in the IaMP is characterized by the product of the information index of the asset and its weight in the market portfolio. With this new benchmark portfolio, we analytically derive the Information-adjusted CAPM (IaCAPM), which states that the expected return on a risky asset/portfolio is a linear function of its information-adjusted beta, with a slope equal to the expected excess return on the IaMP and an intercept equal to the riskless interest rate. The information-adjusted beta measures the systematic risk of an asset and is defined as the covariance between returns on the asset and the IaMP divided by the variance of return on the IaMP. The IaMP plays a similar role as the market portfolio in the classic CAPM and the IaCAPM remains a single-

\footnotetext{
${ }^{2}$ The Sharpe-Lintner-Black CAPM is referred to as the classic CAPM in this paper.

3 The information index is formally defined as the ratio of the unconditional precision of a security's return to the mean of the conditional precision of the return. The details are given in Section III.
} 
factor market model. Nevertheless, the factor that causes systematic return differences across assets is the information-adjusted beta.

The IaCAPM implies that in empirical tests of an asset pricing model, the regression of return should be on information-adjusted beta rather than market beta in cross-sectional analysis, or on excess return on the IaMP rather than excess market return in time-series analysis. When market beta or excess market return is chosen as an explanatory variable, the model essentially turns out to be a random coefficient regression model. This is likely to be one of main reasons for various anomalies discovered in the empirical tests of the classic CAPM. Proposition 4 in the paper specifies the conditions under which the Sharpe-Lintner CAPM overstates or understates the expected return on a risky security or a portfolio of securities, and quantify the prediction error. Based on this proposition, Section IV analyzes the possible reasons for the empirical anomalies discovered in the literature.

We adopt the approach of rational expectations analysis to relax the assumption of complete agreement. Following the pioneering works of Grossman (1976), and Grossman and Stiglitz (1980), rational expectations models developed by, for example, Hellwig (1980), Admati (1985), Easley and O'Hara (2004), focus on the effect of asymmetric information on capital asset pricing. They usually assume that the prices of risky assets and signals conveying price information are normally distributed random variables. The normal distributions, accompanied by constant absolute risk aversion utility, can generate elegant linear demand functions and a closed-form solution for conditional expectations of relevant variables. Although they have been successfully used to demonstrate that the conditional CAPM (i.e., expectations are taken conditional on investors' posterior beliefs) holds in various variants (e.g., Admati (1985)), they can hardly be applied to the analysis of unconditional expected return. ${ }^{4}$ The reason is that

\footnotetext{
${ }^{4}$ It is important to distinguish unconditional expectation from conditional expectation. Equilibrium prices of risky assets depend on a particular realization of random informative signals and random asset supplies in a one-period model. So, conditional on the realization of signals and supplies, the randomness of return on a risky asset stems solely from the randomness of the future value (or future price) of the asset. For unconditional expected return, however, the expectation operation should be taken over all random variables and in turn the equilibrium prices should be considered as random too. See, for example, Easley and O'Hara (2004).
} 
the assumption of normally distributed prices ultimately results in a distribution of returns for which unconditional expectation does not exist. ${ }^{5}$ To avoid this difficulty, Easley and O'Hara (2004) focus on the analysis of unconditional expected return per share instead of the rate of return. (Throughout the paper, return means the rate of return unless otherwise specified.)

The importance of developing an asset pricing model based on unconditional expectations is twofold. First, as Jagannathan and Wang (1996) and Campbell (2000) have pointed out, even if the conditional CAPM is assumed to be true the return-beta relationship in the classic CAPM usually does not hold when expectations are taken unconditionally. Secondly, an equilibrium based on conditional expectations depends on a particular realization of random parameters and variables. But most empirical tests, which disclose various anomalies of the classic CAPM as represented by Fama and French (1992, 1993), implicitly require an asset pricing model established on unconditional expectations. ${ }^{6}$ In order to develop an unconditional asset pricing model and circumvent the difficulty caused by the normal distribution assumption, we extend the Easley-O'Hara (2004) model by adopting lognormal distributions of asset values and informative signals. We show that although the conditional CAPM does hold in our framework, as found by other asset pricing models of rational expectations equilibrium, the unconditional CAPM has to be modified to accommodate belief diversity among investors.

The rest of the paper is organized as the follows. Section II specifies the Bayesian belief updating process based on lognormally distributed signals. Then it completes an asymmetric information model by establishing rational expectations equilibrium. Section III calculates conditional and unconditional expected returns in equilibrium, and derives the IaCAPM by dropping the assumption of complete

\footnotetext{
${ }^{5}$ Simple return involves the ratio of random future value to random equilibrium price and its unconditional expectation is not computable because even for two independent standard normal variables their ratio follows a Cauchy distribution, for which both mean and variance do not exist (Hogg and Craig (1995), p. 174). Continuously compounded return is not well-defined due to the negative range of normal variates.

${ }^{6}$ Of course, empirical test methods for conditional asset pricing models have been well developed; see for instance, Cochrane (2005).
} 
agreement. It also discusses the main properties and predictions of the IaCAPM. Section IV analyzes the anomalies documented in the empirical analysis of the classic CAPM from the IaCAPM perspective. Concluding remarks are given in the final section. Proofs of propositions and corollaries are given in Appendix A.

\section{Belief Updating and Rational Expectations Equilibrium}

\section{A. Bayesian Inference}

Consider a market of $K$ risky assets and one risk-free asset. Agents in this market optimize their asset portfolios through trading securities at date 0 to maximize their expected utility at date 1. Although nobody knows the future values of risky assets, $v_{k}(k=1, \ldots, K)$, before trading, their prior distributions are common knowledge. It is assumed that $v_{k}$ are independently, lognormally distributed, ${ }^{7}$ i.e.,

$$
\ln v_{k} \sim N\left(\overline{\ln v_{k}}, \rho_{k}^{-1}\right), \quad k=1, \ldots, K .
$$

There are $I_{k}$ signals revealing the information of the future value of risky asset $k$ before trading. These signals, $s_{k 1}, s_{k 2}, \ldots, s_{k I_{k}}$, are drawn independently from a lognormal distribution, conditional on a realization of $v_{k}$, i.e.,

$$
\ln s_{k j} \mid v_{k} \sim N\left(\ln v_{k}, \gamma_{k}^{-1}\right), \quad j=1, \ldots, I_{k} .
$$

Some of these signals are public information observed by all investors in the market but the others are private and observed only by a portion of investors. The fraction of private signals is denoted by $\alpha_{k}$ so that the fraction of signals publicly observable is $1-\alpha_{k}$. Investors who can observe both private and public signals of asset $k$ are called informed investors, whereas investors who only observe public signals are called uninformed investors. $^{8}$ To facilitate analysis and simplify notations, we define two statistics of these signals:

\footnotetext{
${ }^{7}$ The assumption of independent distributions of asset values has also been adopted by other studies of asset pricing; for instance, Easley and O'Hara (2004) and O'Hara (2003). The introduction of correlations between risky assets does not change the core result of the paper-the IaCAPM and its predictions. Appendix B to this paper, which is available upon request, outlines the derivation of the IaCAPM and other main results when all risky assets and signals are correlated. However, the assumption of independent distributions can greatly simplify algebra and ease the exposition of economic intuition. So, we keep it throughout the main text.

${ }^{8}$ An investor informed of asset $k$ can be uninformed of asset $j$ if he/she does not receive the private signals of asset $j$.
} 


$$
g_{k} \equiv \sum_{i=1}^{\alpha_{k} I_{k}} \ln s_{k i} / \alpha_{k} I_{k}, \quad h_{k} \equiv \sum_{i=\alpha_{k} I_{k}+1}^{I_{k}} \ln s_{k i} /\left(1-\alpha_{k}\right) I_{k} .
$$

It can be easily verified that $g_{k}$ and $h_{k}$ are normal, conditional on $v_{k}$, that

$$
\begin{array}{ll}
g_{k} \mid v_{k} \sim N\left(\ln v_{k},\left(\gamma_{k} \alpha_{k} I_{k}\right)^{-1}\right), & k=1, \ldots, K, \\
h_{k} \mid v_{k} \sim N\left(\ln v_{k},\left[\gamma_{k}\left(1-\alpha_{k}\right) I_{k}\right]^{-1}\right), & k=1, \ldots, K .
\end{array}
$$

Since $g_{k}$ and $h_{k}$ are sufficient statistics for the collections of private signals and public signals, respectively, we only need to investigate these two sufficient statistics, instead of considering signals $s_{k 1}, s_{k 2}, \ldots, s_{k I_{k}}$ individually.

Informed investors can use both private information $g_{k}$ and public information $h_{k}$ to update their beliefs on the future values of risky assets. Thus, applying Bayes' rule they have the following posterior belief on asset $k$ :

$$
\ln v_{k}^{i} \mid\left(g_{k}, h_{k}\right) \sim N\left(\frac{\ln _{k}}{I},\left(\lambda_{k}^{I}\right)^{-1}\right), \quad \text { if inventor } i \text { is informed, }
$$

where the conditional mean and conditional precision are given by

$$
\begin{aligned}
& \overline{\ln v}_{k}^{I} \equiv\left[\rho_{k} \overline{\ln v_{k}}+\gamma_{k} \alpha_{k} I_{k} g_{k}+\gamma_{k}\left(1-\alpha_{k}\right) I_{k} h_{k}\right] / \lambda_{k}^{I}, \\
& \lambda_{k}^{I} \equiv \rho_{k}+\gamma_{k} I_{k} .
\end{aligned}
$$

Uninformed investors only receive public information, $h_{k}$. But the equilibrium prices of risky assets partially reveal the information contained in private signals. So, they use both $h_{k}$ and price signals to update their beliefs. Let $p_{k}$ be the equilibrium price of risky asset $k$. Assume that the supply of the $k^{\text {th }}$ asset in terms of its total value, $y_{k}$, is a normal random variable, independent of other random variables, with mean $\bar{y}_{k}>0$ and precision $\eta_{k} \cdot{ }^{9}$ Suppose that uninformed investors conjecture the following equilibrium price (it will be verified later that this conjecture is self-fulfilling):

$$
\ln p_{k}=B_{v} \overline{\ln v_{k}}+B_{g} g_{k}+B_{h} h_{k}-B_{y} y_{k}+B_{\bar{y}} \bar{y}_{k},
$$

where $B_{v}, B_{g}, B_{h}, B_{y}$ and $B_{\bar{y}}$ are constants and will be given in Proposition 1 below. ${ }^{10}$ To facilitate analysis, we define a random variable that

\footnotetext{
${ }^{9}$ It is well-known that the market must have some noise to avoid a perfectly revealing equilibrium. Asymmetric information models (e.g., Admati (1985), Easley and O'Hara (2004)) typically assume that the number of per-capita supply of a stock is a normal random variable. Since the supply randomness is usually considered as the result of trading by liquidity traders who buy or sell an asset for their liquidity purposes, regardless of its price, assuming random total supply value seems reasonable if it is not more plausible. The assumption $\bar{y}_{k}>0$ implies a positive supply on average.

${ }^{10}$ To ease exposition, we have dropped subscript $k$ in $B_{v}, B_{g}, B_{h}, B_{y}$ and $B_{\bar{y}}$.
} 


$$
\theta_{k} \equiv\left[\ln p_{k}-B_{v} \overline{\ln v_{k}}-B_{h} h_{k}+\bar{y}_{k}\left(B_{y}-B_{\bar{y}}\right)\right] / B_{g}=g_{k}-\left(y_{k}-\bar{y}_{k}\right) B_{y} / B_{g} .
$$

Since uninformed investors can compute $\theta_{k}$ by price and public information, observing signal $\theta_{k}$ is equivalent to observing price signal $p_{k}$. It is clear that

$$
\begin{aligned}
& E\left(\theta_{k} \mid v_{k}\right)=E\left(g_{k} \mid v_{k}\right)-E\left[\left(y_{k}-\bar{y}_{k}\right) B_{y} / B_{g}\right]=\ln v_{k}, \\
& \lambda_{\theta k} \equiv\left[\operatorname{var}\left(\theta_{k} \mid v_{k}\right)\right]^{-1}=\left[\left(B_{y} / B_{g}\right)^{2} \eta_{k}^{-1}+\left(\gamma_{k} \alpha_{k} I_{k}\right)^{-1}\right]^{-1} .
\end{aligned}
$$

Thus, $\theta_{k} \mid v_{k} \sim N\left(\ln v_{k}, \lambda_{\theta k}^{-1}\right)$. From public information and signal $\theta_{k}$, the uninformed investors form their posterior beliefs on asset $k$ as:

$$
\ln v_{k}^{i} \mid\left(\theta_{k}, h_{k}\right) \sim N\left(\overline{\ln }_{k}^{U},\left(\lambda_{k}^{U}\right)^{-1}\right), \quad \text { if inventor } i \text { is uninformed. }
$$

In (10), the conditional mean and conditional precision can be expressed as

$$
\begin{aligned}
& \overline{\ln v_{k}^{U}} \equiv\left[\rho_{k} \overline{\ln v_{k}}+\gamma_{k}\left(1-\alpha_{k}\right) I_{k} h_{k}+\lambda_{\theta k} \theta_{k}\right] / \lambda_{k}^{U}, \\
& \lambda_{k}^{U} \equiv \rho_{k}+\gamma_{k}\left(1-\alpha_{k}\right) I_{k}+\lambda_{\theta k} .
\end{aligned}
$$

As expected, (6) and (11) show that the posterior mean is a weighted average of prior mean and signals' means while (7) and (12) demonstrate that the posterior precision is the sum of prior precision and signals' precisions.

\section{B. Investors' Optimal Portfolios}

Let investor $i$ 's initial wealth endowment be $m^{i}$. If he/she allocates $x_{k}^{i}$ dollars to asset $k(k=1,2, \ldots, K)$ and $x_{f}^{i}$ dollars to the risk-free asset at date 0 , his/her wealth at date 1 is

$$
w^{i}=m^{i}+\sum_{k=1}^{K} x_{k}^{i}\left(\frac{v_{k}}{p_{k}}-1\right),
$$

where the risk-free asset is assumed to be the numeraire and has a zero return. To compute investors' demands for assets, we assume that they have identical utility with constant absolute risk aversion, $u(w)=-\exp (-\delta w)$, where $\delta>0$ is the Arrow-Pratt measure of absolute risk aversion. Substituting (13) into the utility function, the expected utility of investor $i$ can be expressed as

$$
E\left[u\left(w^{i}\right) \mid \Pi^{i}\right] \approx-e^{-\delta m^{i}} \exp \left[-\delta \sum_{k=1}^{K} x_{k}^{i}\left(\overline{\ln v}_{k}^{i}-\ln p_{i}-\delta x_{k}^{i} / 2 \lambda_{k}^{i}\right)\right],
$$


where $\Pi^{i}$ is the information set of investor $i$ at the time he/she is making investment decision. ${ }^{11}$ In deriving (14), we have noticed that the return on asset $k, r_{k} \equiv \ln \left(v_{k} / p_{k}\right)$, is conditionally normal so that (5) and (10) imply

$$
E\left(\ln v_{k} \mid \Pi^{i}\right)-\ln p_{k}=\overline{\ln }_{k}^{i}-\ln p_{k}, \quad \quad \operatorname{var}\left(\ln v_{k} \mid \Pi^{i}\right)=\left(\lambda_{k}^{i}\right)^{-1} .
$$

Thus, the maximization of (14) yields the optimal investment strategy for investor $i$ :

$$
x_{k}^{i}=\lambda_{k}^{i}\left(\overline{\ln v}_{k}^{i}-\ln p_{k}\right) / \delta, \quad k=1, \ldots, K, \quad x_{f}^{i}=m^{i}-\sum_{k=1}^{K} x_{k}^{i} .
$$

In (15), $\overline{\ln v}_{k}^{i}$ and $\lambda_{k}^{i}$ are determined by (6)-(7) or (11)-(12), depending on whether investor $i$ receives private signals of asset $k$ or not. The optimal investment (15) is well behaved and has the properties as expected. It shows that an investor invests more in an asset as the expectation of the logarithm of its future value increases and/or the uncertainty becomes smaller. The investment also increases when the conditional expected return, $\overline{\ln v}_{k}^{i}-\ln p_{k}$, is higher. However, when the price of an asset rises, the relative investment in this particular asset declines. As the investor is more risk-averse, he/she reduces his/her investment across all risky assets and increases his/her holding of the risk-free asset. By simple manipulation, we find that investor $i$ choose the following optimal portfolio of risky assets:

$$
t_{k}^{i}=\zeta^{i} \lambda_{k}^{i}\left(\overline{\ln v}_{k}^{i}-\ln p_{k}\right), \quad k=1, \ldots, K,
$$

where $\zeta^{i} \equiv\left(\sum_{k=1}^{K} \lambda_{k}^{i}\left(\overline{\ln v_{k}}-\ln p_{k}\right)\right)^{-1}$ is the normalization coefficient.

\section{Rational Expectations Equilibrium}

Normalize the total number of investors to 1 , with $\kappa_{k}$ investors observing both private and public signals of asset $k$ and $1-\kappa_{k}$ investors observing public signals only $(k=1, \ldots, K)$. We consider rational expectations equilibrium; i.e., each investor's expectation is self-fulfilling in equilibrium. The market clearing condition requires the aggregate demand for each asset equal to its aggregate supply, i.e.,

\footnotetext{
${ }^{11}$ Following the approximation approaches in Biais (1993), Stoll (1978), and Huang and Litzenberger (1988), a Taylor's expansion to utility is used in (14). The residual of this expansion and its effect on utility are second-order small. The proof (Appendix C) is available upon request. Intuitively, the approximation is equivalent to approximating simple return by continuously compounded return.
} 


$$
\kappa_{k} x_{k}^{I}+\left(1-\kappa_{k}\right) x_{k}^{U}=y_{k}, \quad k=1, \ldots, K .
$$

Recalling (6), (7), (11) and (12), we obtain the equilibrium prices after inserting optimal investment in (15) into (17). This is summarized in Proposition 1 below.

PrOPOSITION 1. There exists a partially revealing rational expectations equilibrium in which,

$$
\ln p_{k}=B_{v} \overline{\ln v_{k}}+B_{g} g_{k}+B_{h} h_{k}-B_{y} y_{k}+B_{\bar{y}} \bar{y}_{k}, \quad k=1, \ldots, K,
$$

where coefficients B's are given in the proof of the proposition in Appendix A.

The price formula in equation (18) has a standard form, the fundamental value of the security less the supply pressure. The fundamental value consists of three components, the prior value $\left(\overline{\ln v_{k}}\right)$, the private $\left(g_{k}\right)$ and public $\left(h_{k}\right)$ signals. They are incorporated into the equilibrium price in a form of weighted average as the weights, $B_{v}, B_{g}$ and $B_{h}$ add up to one. The supply pressure component, $-\left(B_{y} y_{k}-B_{\bar{y}} \bar{y}_{k}\right)$, represents the price discount the investors require to hold the risky security. The larger the supply $y_{k}$ relative to its mean $\bar{y}_{k}$, the larger the discount.

\section{The Information-adjusted CAPM}

\section{A. Expected Returns}

Conditional on his/her information, investor $i$ 's expected return on asset $k$ is

$$
\bar{r}_{k}^{i} \equiv\left\{\begin{array}{ll}
\bar{r}_{k}^{I}=E\left(r_{k} \mid \Pi^{I}\right)=\overline{\ln }_{k}^{I}-\ln p_{k} & \text { if } i \text { is informed } \\
\bar{r}_{k}^{U}=E\left(r_{k} \mid \Pi^{U}\right)=\overline{\ln v}_{k}^{U}-\ln p_{k} & \text { if } i \text { is uninformed }
\end{array} .\right.
$$

The corresponding precisions are $\lambda_{k}^{I}$ and $\lambda_{k}^{U}$, respectively, for informed and uninformed investors. Therefore, an investor with the private information of asset $k$ believes that the return follows a normal distribution $N\left(\bar{r}_{k}^{I},\left(\lambda_{k}^{I}\right)^{-1}\right)$ whereas an uniformed investor believes the distribution is $N\left(\bar{r}_{k}^{U},\left(\lambda_{k}^{U}\right)^{-1}\right)$. Since there are $\kappa_{k}$ investors with information precision $\lambda_{k}^{I}$ and $1-\kappa_{k}$ investors with precision $\lambda_{k}^{U}$, the average conditional belief, following Lintner (1969), and Easley and O'Hara (2004), can be defined as,

$$
\begin{aligned}
& \bar{r}_{k}^{M} \equiv\left[\lambda_{k}^{I} \kappa_{k} \bar{r}_{k}^{I}+\lambda_{k}^{U}\left(1-\kappa_{k}\right) \bar{r}_{k}^{U}\right] / \lambda_{k}^{M}, \\
& \lambda_{k}^{M} \equiv \lambda_{k}^{I} \kappa_{k}+\lambda_{k}^{U}\left(1-\kappa_{k}\right) .
\end{aligned}
$$


Because investors make their investment decision by maximizing their conditional mean-variance utility, it is obvious that the risky portfolio $\left\{t_{k}^{i}\right\}$ as characterized by (16) is the tangency portfolio of investor $i$, according to his/her posterior belief of returns on risky assets. Moreover, applying the result of conditional mean-variance efficiency of each investor's investment decision it can be easily shown that the Sharpe-Lintner CAPM conditionally holds for the average conditional belief in (20)-(21); that is, the conditional expectation of excess return on a portfolio of risky assets is equal to the conditional expectation of market excess return times the conditional market beta of the portfolio. A similar result is also obtained by other rational expectations models such as Admati (1985). However, the main interest of this paper is to develop an asset pricing model that holds unconditionally. For this end, we first need to find out the unconditional expected returns on all individual risky assets, which will be given in Proposition 2 below. Secondly, we have to determine what factors summarize the systemic risk of an asset or a portfolio. To deal with this, we, following the classic CAPM, identify a portfolio in the next subsection that captures the supply and information characteristics of the entire market so that it plays the same role as the market portfolio does in the classic CAPM.

Proposition 2. The unconditional expected value and precision of return $r_{k}$ on asset $k$ $(k=1, \ldots, K)$ are given by

$$
\begin{aligned}
& \bar{r}_{k}=\frac{\delta \bar{y}_{k}}{\rho_{k}+\gamma_{k}\left(1-\alpha_{k}\right) I_{k}+\kappa_{k} \gamma_{k} \alpha_{k} I_{k}+\left(1-\kappa_{k}\right) \xi_{k}}=\frac{\delta \bar{y}_{k}}{\lambda_{k}^{M}}, \\
& \lambda_{k}=\left[B_{v}^{2} / \rho_{k}+B_{g}^{2} / \gamma_{k} \alpha_{k} I_{k}+B_{h}^{2} / \gamma_{k}\left(1-\alpha_{k}\right) I_{k}+B_{y}^{2} / \eta_{k}\right]^{-1} .
\end{aligned}
$$

Proposition 2 says that the unconditional expected return is the product of risk aversion $(\delta)$, riskiness $\left(\left(\lambda_{k}^{M}\right)^{-1}\right)$ and the mean of supply $\left(\bar{y}_{k}\right)$. It increases as the investors become more risk averse (larger $\delta$ ), the payoff becomes riskier ( $\operatorname{smaller} \lambda_{k}^{M}$ ), and/or they have to accommodate more supply on average (larger $\bar{y}_{k}$ ). The unconditional variance of the return is a weighted sum of the prior variance of asset value $\left(\rho_{k}^{-1}\right)$, and the variances of private signals $\left(\left(\alpha_{k} I_{k} \gamma_{k}\right)^{-1}\right)$, public signal $\left(\left(\left(1-\alpha_{k}\right) I_{k} \gamma_{k}\right)^{-1}\right)$ and security supply $\left(\eta_{k}^{-1}\right)$. 


\section{B. The Information-adjusted CAPM}

Once the assumption of complete agreement is relaxed, the information structure of a market is vital. Two parameters responsible for the characteristics of information in the market are conditional and unconditional precisions, $\lambda_{k}^{M}$ and $\lambda_{k}$, respectively. Conditional precision $\lambda_{k}^{M}$ reflects the accuracy of the knowledge about the future value of stock $k$ a posteriori once signals are received but does not capture the variability due to different realizations of signals. On the other hand, unconditional precision $\lambda_{k}$ reflects the total precision of the future value but does not reveal how much information can be extracted from a particular realization of signals in statistical inference. Therefore, we define information index as the ratio of unconditional precision to conditional precision, $\omega_{k} \equiv \lambda_{k} / \lambda_{k}^{M}$, to depict the effective information about asset $k$ possessed by investors. For a risky portfolio $\left\{t_{k}\right\}$, the information index is defined as $\omega_{P} \equiv\left(\sum_{k=1}^{K} \pi_{k} / \lambda_{k}^{M}\right) /\left(\sum_{k=1}^{K} \pi_{k} / \lambda_{k}\right)$, where $\pi_{k}=t_{k} \bar{y}_{k} / \sum_{j=1}^{K} t_{j} \bar{y}_{j} \quad(k=1, \ldots$, $K)$. Clearly, $\omega_{P}$ reduces to $\omega_{k}$ if the portfolio includes risky asset $k$ only. The justification for the information index as a suitable measure of the amount of information on a security or a portfolio can be further seen from Lemma 1 in the next section. It attains a maximum of 1 (or a minimum of 0 ) if the amount of the information (or, more precisely, the total information quality as defined in Lemma 1) is infinite (or zero).

Based on the information index, we can introduce the information-adjusted market portfolio to incorporate information effects. Since the average market supply of assets $k$ is $\bar{y}_{k}(k=1, \ldots, K)$, each weight in the market portfolio is given by $t_{k}^{M} \equiv \zeta_{M} \bar{y}_{k}$, where $\zeta_{M} \equiv\left(\sum_{k=1}^{K} \bar{y}_{k}\right)^{-1}$ is the normalization constant. Consequently, the IaMP, $\left\{t_{k}^{A}\right\}$, is defined as the market portfolio adjusted for the information index; i.e., $t_{k}^{A}$ is equal to $t_{k}^{M} \omega_{k}$, up to a normalization constant. By choosing the normalization constant $\zeta_{A} \equiv\left(\sum_{k=1}^{K} \bar{y}_{k} \omega_{k}\right)^{-1}$, we obtain $t_{k}^{A} \equiv \zeta_{A} \bar{y}_{k} \omega_{k}$. Generally, an asset with more (less) information revealed to the market has a larger (smaller) weight in the IaMP than in the 
market portfolio. The definition of the IaMP captures the aggregate characteristics of the market: the distributions of both market supply and information index across securities.

Proposition 3. Under the assumptions of the modeled market, the following IaCAPM

$$
\bar{r}_{k}=\bar{r}_{A} \beta_{k}^{A}, \quad k=1,2, \ldots, K,
$$

holds, where $\bar{r}_{A} \equiv \sum_{k=1}^{K} t_{k}^{A} \bar{r}_{k}$ is the unconditional expected return on the IaMP, $\beta_{k}^{A} \equiv \sigma_{k A} / \sigma_{A A}$ is the information-adjusted beta, $\sigma_{k A}=t_{k}^{A} / \lambda_{k}$ is the covariance between returns on asset $k$ and on the IaMP, and $\sigma_{A A}=\sum_{k=1}^{K}\left(t_{k}^{A}\right)^{2} / \lambda_{k}$ is the variance of return on the IaMP. The IaMP is mean-variance efficient with respect to mean $\bar{r}_{k}$ and precision $\lambda_{k}$. For a portfolio $\left\{t_{k}\right\}$, the IaCAPM also holds that

$$
\bar{r}_{P}=\bar{r}_{A} \beta_{P}^{A},
$$

where $\bar{r}_{P}=\sum_{k=1}^{K} t_{k} \bar{r}_{k}$ and $\beta_{P}^{A}=\sum_{k=1}^{K} t_{k} \beta_{k}^{A}$ are the unconditional expected return and the information-adjusted beta of the portfolio.

\section{Remarks on the Information-adjusted CAPM}

1. It is the information-adjusted beta, rather than the market beta, that can solely explain the systematic variation in returns and no other factor is required to explain the variation. The unconditional expected excess return on an asset is proportionate to its information-adjusted beta, and the beta premium is positive and equal to the unconditional expected excess return on the IaMP.

2. The essential difference between the IaCAPM and the Sharpe-Lintner CAPM is that the market portfolio and market beta are replaced by the IaMP and informationadjusted beta. In the Sharpe-Lintner CAPM, investors are symmetric and are supposed to know the true joint distribution of returns. Under this information environment, all investors hold an identical portfolio in equilibrium. Consequently, the systematic variation in the return on an individual asset is completely captured by its covariance with the market return. Nevertheless, when investors do not have complete agreement on asset returns, investors in the market have to collect information to update their beliefs. This results in diverse information indexes. The 
covariance of the return on a security with the market return, or the market beta, cannot capture this information effect on the market and in turn the market beta cannot properly reveal the systematic risk a stock. Instead, Proposition 3 asserts that the systematic variation of returns is jointly captured by the information index and market capitalization, or more precisely, the information-adjusted beta.

3. The IaCAPM established in Proposition 3 is empirically testable. The difference between the tests of the IaCAPM and the classic CAPM is the former needs information index to construct the IaMP. By definition, information index $\omega_{k}$ is equal to $\lambda_{k} / \lambda_{k}^{M}$, where $\lambda_{k}$ and $\lambda_{k}^{M}$ are constant and do not include any state variables or information signals received by investors. Since $\lambda_{k}$ is the inverse of the unconditional variance of return, it can be easily estimated by conventional methods. On the other hand, $\lambda_{k}^{M}$ may be estimated by high frequency return data. Therefore, econometricians would be able to empirically test the IaCAPM ex post without knowing observations available only to market participants or utilizing macroeconomic variables revealing the state of the market.

4. The importance of the IaMP can also be seen from its mean-variance efficiency with respect to the belief $\left(\bar{r}_{k}, \lambda_{k}\right)$, as indicated in Proposition 3. Note that Easley and O'Hara (2004) shows that the market portfolio is mean-variance efficient with respect to mean $\bar{r}_{k}$ and precision $\lambda_{k}^{M}$ although the return in their model is defined as return per share. It is not hard to demonstrate that the market portfolio in our model has a similar property. However, the unconditional mean $\bar{r}_{k}$ and conditional precision $\lambda_{k}^{M}$ do not constitute a well-defined belief because it is the pair $\left(\bar{r}_{k}, \lambda_{k}\right)$ rather than the pair $\left(\bar{r}_{k}, \lambda_{k}^{M}\right)$ that forms the unconditional mean and unconditional precision of random variable $r_{k}$.

To illustrate the significance of Proportions 3, let us consider an imaginary market, where all investors are risk averse and have an absolute risk-aversion coefficient of $\delta=1$. There are 25 stocks in the market with means of supplies equal to $\bar{y}_{k}=0.5+k / 25(k=1, \ldots, 25)$. Average conditional precisions of stock returns are set at $\lambda_{k}^{M}=0.1$ while unconditional precisions are determined by $\lambda_{k}=0.01+0.09 U_{k}$, 
where $U_{k}(k=1, \ldots, 25)$ are independent uniform variates on $[0,1]$. Then, the information index can be calculated by definition $\omega_{k}=\lambda_{k} / \lambda_{k}^{M}$ and unconditional expected returns are found by Proportion 2 that $\bar{r}_{k}=10 \bar{y}_{k}$. The weights of the market portfolio and IaMP are determined by their definitions that $t_{k}^{M}=\bar{y}_{k} / \sum_{j=1}^{25} \bar{y}_{j}$ and $t_{k}^{A}=\omega_{k} \bar{y}_{k} / \sum_{j=1}^{25} \omega_{j} \bar{y}_{j}$. Market beta $\beta_{k}^{M}$ and information-adjusted beta $\beta_{k}^{A}$ can be calculated from the market portfolio and IaMP, respectively. Figure 1a below depicts the scatter plot of 25 pairs of market beta-returns $\left(\beta_{k}^{M}, \bar{r}_{k}\right)$ we have generated, which simulates observed market beta-return relationship in a real market and exhibits conventional anomalies well-known in the literature. For instance, the 25 points do not fall on a nominal straight line as predicted by the classical CAPM, indicating other explanatory variables are required to explain the variation of return; the regression line of these 25 stocks is much flatter than the market security line of Sharp-Lintner CAPM. However, if the scatter plot is drawn against information-adjusted beta rather than market beta, then our simulated data show the corresponding 25 pairs of $\left(\beta_{k}^{A}, \bar{r}_{k}\right)$ fall exactly on the straight line of the IaCAPM, as shown in Figure 1b. This example demonstrates that it is the information-adjusted beta, rather than the market beta, that can solely explain the systematic variation in returns.

\section{Figure 1 is about here}

\section{The Inaccuracy of the Predictions of the Sharpe-Lintner CAPM}

Proposition 4. When the information index varies across securities, there exists a unique $\omega^{*} \in(0,1)$ such that the relative prediction error made by the Sharpe-Lintner CAPM on the expected return on a portfolio is proportionate to $\left|\omega_{P}-\omega^{*}\right|$, i.e.

$$
\left|\bar{r}_{P}-\bar{r}_{M} \beta_{P}^{M}\right| /\left|\bar{r}_{M} \beta_{P}^{M}\right|=c\left|\omega_{P}-\omega^{*}\right|
$$

where $c \equiv \bar{r}_{A} \sigma_{M M} \zeta_{A} / \bar{r}_{M} \sigma_{A A} \zeta_{M}$ is a constant across all securities. Moreover, for a portfolio with a positive market beta (i.e., $\left.\beta_{P}^{M}>0\right)$, the Sharpe-Lintner CAPM 
overstates the unconditional expected return on the portfolio if $\omega_{P} \in\left(0, \omega^{*}\right)$, whereas it understates the return if $\omega_{P} \in\left(\omega^{*}, 1\right) .^{12}$

If all $\omega_{k}$ are equal, the IaCAPM collapses to the Sharpe-Lintner CAPM.

Based on the data used in Figure 1, ${ }^{13}$ Table 1 displays the absolute and relative prediction errors of the Sharpe-Lintner CAPM. It can be seen from the upper panel of the table that when $\omega_{k}<\omega^{*}=0.31\left(\omega_{k}>\omega^{*}=0.31\right)$ the prediction errors are negative (positive) so that the Sharpe-Lintner CAPM overstates (understates) unconditional expected returns. The lower panel of the table demonstrates the conclusion of Proposition 4 that the relative error made by the Sharpe-Lintner CAPM increases monotonically and is proportionate to $\left|\omega_{P}-\omega^{*}\right|$.

\section{Table 1 is about here}

\section{Why Does the Classic CAPM Fail in Empirical Tests?}

The IaCAPM suggests that empirical tests should be carried out by regressing excess return on information-adjusted beta in cross-sectional analysis or on the risk premium of the IaMP in time-series analysis. When regressions use market beta or market excess return as the only explanatory variable, they fail to capture the crosssectional variability in information quality, as indicated in Proposition 4. This may be partially responsible for various anomalies discovered in the existing tests of the classic CAPM. This section details the IaCAPM's explanation to these anomalies.

\section{A. Why Is the Slope of Empirical Relation between Market Beta and Average Return So}

Flat?

The first anomaly we are going to examine is the empirical finding of the flatter slope, i.e., beta premium obtained in cross-sectional analysis is much smaller than the

\footnotetext{
${ }^{12}$ There are other sufficient conditions for the prediction of the Sharpe-Lintner CAPM overstating or understating returns. But only considering these two scenarios is enough for our purpose because market betas and weights of portfolios are generally positive in empirical studies cited in the next section.

${ }^{13} \mathrm{An}$ additional ingredient is $\omega^{*}$, which is equal to 0.31 according to the calculation shown in the proof of Proposition 4.
} 
average excess market return (see, Black, Jensen and Scholes (1972), and Fama French (1992)). To facilitate the analysis, let us introduce a measure of the absolute information quality of a risky security, i.e., the total information precision of asset $k$, $\Psi_{k} \equiv \rho_{k}+\gamma_{k} I_{k}$. Correspondingly, the information quality of a portfolio is defined by a harmonic average of individual qualities, $\Psi_{P} \equiv\left(\sum_{k=1}^{K}\left|\pi_{k}\right| \Psi_{k}^{-1}\right)^{-1}$. There is a close relationship between the information index and information quality, as demonstrated by the lemma below.

LEMMA 1. For each asset, information index $\omega_{k}$ and information quality $\Psi_{k}$ have the following properties: (i) $\omega_{k} \rightarrow 1$ and $\lambda_{k} \rightarrow+\infty$ if $\Psi_{k} \rightarrow+\infty$ and $\lim _{\substack{\gamma_{k} I_{k} \rightarrow+\infty \\ \rho_{k} \rightarrow+\infty}} \rho_{k} / \gamma_{k} I_{k}$ exists; ${ }^{14}$ (ii) $\omega_{k} \rightarrow 0$ and $\lambda_{k} \rightarrow 0$ if $\Psi_{k} \rightarrow 0$; (iii) $0 \leq \omega_{k} \leq 1$. For a portfolio, the corresponding properties are: (iv) $\omega_{P} \rightarrow 1$ if $\Psi_{P} \rightarrow+\infty$ and $\lim _{\substack{\gamma_{\rho_{k}} I_{k} \rightarrow+\infty \\ \rho_{k} \rightarrow+\infty}} \rho_{k} / \gamma_{k} I_{k}$ exist for all $k$; (v) $\omega_{P} \rightarrow 0$ if $\Psi_{P} \rightarrow 0$; (vi) $0 \leq \omega_{P} \leq 1$ if $t_{k} \geq 0$ for all $k$.

To see why the empirical line is flatter, we rewrite the IaCAPM as

$$
\bar{r}_{P}=c_{P} \bar{r}_{M} \beta_{P}^{M},
$$

where $c_{P} \equiv c \omega_{P}$ and constant $c$ is given in Proposition 4. Since in empirical studies market betas and unconditional expected returns are positive, if we can demonstrate that the value of $c_{P}$ tends to be greater and smaller than 1 for small and large $\beta_{P}^{M}$, respectively, the flatter slope puzzle is, at least partially, resolved. Recall that Proposition 4 specifies two distinct areas of information index $\omega_{P}$, where the SharpeLintner CAPM overstate or understate expected return. Thus, using the link between information index $\omega_{P}$ and information quality $\Psi_{P}$ established in Lemma 1, we can easily determine the condition for the Sharpe-Lintner CAPM overstating or understating the expected return in Corollary 1 via the bridge of Proposition 5 below.

Proposition 5. There exist two constants, $\Psi_{L}$ and $\Psi_{U}\left(0<\Psi_{L}<\Psi_{U}<+\infty\right)$, such that (i) the Sharpe-Lintner CAPM overstates the expected return on a portfolio (i.e., $\left.c_{P}<1\right)$ if the portfolio's information quality $\Psi_{P}$ is low and within $\left[0, \Psi_{L}\right)$;

\footnotetext{
${ }^{14}$ This includes the existence of an infinite limit.
} 
(ii) the Sharpe-Lintner CAPM understates the expected return on a portfolio (i.e., $\left.c_{P}>1\right)$ if the portfolio's information quality $\Psi_{P}$ is high and within $\left(\Psi_{U},+\infty\right)$.

According to Proposition 5, we can define two sets of portfolios $P_{L} \equiv\left\{\right.$ portfolios with $\left.\Psi_{P}<\Psi_{L}\right\}$ and $P_{U} \equiv\left\{\right.$ portfolios with $\left.\Psi_{P}>\Psi_{U}\right\} . \quad$ Thus, another way to express Proposition 5 is that the expected returns on portfolios in $P_{L}$ are overstated by the Sharpe-Lintner CAPM whereas the expected returns on portfolios in $P_{U}$ are understated. Because portfolios in set $P_{L}$ tend to have a large market beta while their counterparts in $P_{U}$ tend to have a small market beta, the following corollary connects the over- or under-statement of the Sharpe-Lintner CAPM with the market beta.

COROLlaRY 1. There exists a constant, $\beta_{U}$, such that $\beta_{P}^{M} \in\left(\beta_{U},+\infty\right)$ for all portfolios in set $P_{L}$; that is, portfolios whose unconditional expected returns are overstated by the Sharpe-Lintner CAPM tend to have a large market beta. There also exists a constant, $\beta_{L}$, such that $\beta_{P}^{M} \in\left[0, \beta_{L}\right)$ for all portfolios in $P_{U}$; that is, portfolios whose expected returns are understated by the Sharpe-Lintner CAPM tend to have a small market beta.

B. Why Does the Effect of Market Beta on the Average Return Tend to Be Statistically Insignificant?

The classic CAPM claims that the beta premium is positive. Statistically, this claim is valid if the null hypothesis,

$$
\mathrm{H}_{0}: \quad b_{\beta} \leq 0
$$

can be rejected in the following cross-sectional analysis

$$
r_{P}=a+b_{\beta} \beta_{P}^{M}+\varepsilon_{P}, \quad \text { with } \varepsilon_{P} \sim N\left(0, \sigma_{P}^{2}\right)
$$

for pre-selected portfolios $P(P=1,2, \ldots, N)$. Note that the corresponding $t$-test statistic for testing the above null hypothesis is

$$
t=\frac{\hat{b}_{\beta}\left\{\sum_{j=1}^{N}\left(\beta_{j}^{M}-\sum_{i=1}^{N} \beta_{i}^{M} / N\right)^{2}\right\}^{1 / 2}}{\left\{\operatorname{var}\left(r_{P}\right)\right\}^{1 / 2}},
$$


where the variance of return, $\operatorname{var}\left(r_{P}\right)$, is equal to $\sigma_{P}^{2}$ if the slope $b_{\beta}$ in (27) is assumed to be constant across portfolios, as done in the existing cross-sectional analysis, and $\hat{b}_{\beta}$ is the least squares estimate of $b_{\beta}$.

Unfortunately, finance literature has documented that the data collected for empirical analysis are often unable to reject the above null hypothesis (see, for instance, Fama and French, 1992, p. 428). To understand why the null hypothesis of $b_{\beta} \leq 0$ cannot be rejected in theory, let us return to equation (26). According to it, observed data on return and market beta in cross-sectional analysis do not follow a regression equation with a constant slope as assumed by (27). Rather, the slope in (26), $c_{P} \bar{r}_{M}$, varies across portfolios. Hence, if the explanatory variable is market beta rather than information-adjusted beta, a random-coefficient regression model is more appropriate than a constant slope model as implied by the classic CAPM. Such a randomcoefficient regression model involves a two-level regression. ${ }^{15}$ Level 1 relates the return on portfolio $P$ to its market beta,

$$
\text { Level 1: } \quad r_{P}=a+b_{\beta P} \beta_{P}^{M}+\varepsilon_{P} \quad \text { with } \varepsilon_{P} \sim N\left(0, \sigma_{P}^{2}\right) .
$$

Level 2 postulates a statistical model describing the portfolio-to-portfolio variation of the slope caused by unevenly distributed information across portfolios,

$$
\text { Level 2: } \quad b_{\beta P} \sim F\left(\omega_{P}\right),
$$

where $F\left(\omega_{P}\right)$ is the distribution of the information index. According to this two-level regression model, the actual data on return have a variance greater than $\sigma_{P}^{2}$ since

$$
\operatorname{var}\left(r_{P}\right)=E\left\{\operatorname{var}\left(r_{P} \mid b_{\beta P}\right)\right\}+\operatorname{var}\left\{E\left(r_{P} \mid b_{\beta P}\right)\right\}=\sigma_{P}^{2}+\operatorname{var}\left\{E\left(r_{P} \mid b_{\beta P}\right)\right\}>\sigma_{P}^{2} .
$$

Consequently, it leads to a smaller value of $t$-test statistic in (28). This explain, to some extent, why econometricians often cannot reject the null hypothesis of $b_{\beta} \leq 0$.

\section{Why Is Jensen's Alpha Not Equal to Zero?}

We now turn to time-series regression for a portfolio, $P$,

$$
r_{P t}=a_{P}+\beta_{P}^{M} \bar{r}_{M t}+\varepsilon_{P t}, \quad t=1,2, \ldots
$$

\footnotetext{
${ }^{15}$ See Fitzmaurice, Laid and Ware (2004) for an overview about random-coefficient regression analysis.
} 
where intercept $a_{P}$ should be zero according to the Sharpe-Lintner CAPM. However, the regularity found in time-series regressions of excess portfolio return on excess market return is that the intercepts are positive when portfolios have low market betas but negative when portfolios have high betas (Black, Jensen and Scholes (1972), Fama and French (2004)). The IaCAPM may shed light on this conflict between theory and empirical findings. To demonstrate this, let us rewrite equation (25) as

$$
\bar{r}_{P}=\left[\beta_{P}^{A} \bar{r}_{A}-\beta_{P}^{M} \bar{r}_{M}\right]+\beta_{P}^{M} \bar{r}_{M},
$$

which implies that the intercept in equation (29) is given by

$$
a_{P}=\beta_{P}^{A} \bar{r}_{A}-\beta_{P}^{M} \bar{r}_{M}=\beta_{P}^{M} \bar{r}_{M}\left(c_{P}-1\right),
$$

Hence, applying Proposition 5 and Corollary 1 immediately yields the corollary below:

COROLlaRY 2. The intercept of the time-series regression (29) is

(i) negative and its market beta is large (i.e. $\beta_{P}^{M} \in\left(\beta_{U},+\infty\right)$ ) for a portfolio with low information quality (i.e. $\Psi_{P}<\Psi_{L}$ );

(ii) positive and its market beta is small (i.e. $\beta_{P}^{M} \in\left[0, \beta_{L}\right)$ ) for a portfolio with high information quality (i.e. $\Psi_{P}>\Psi_{L}$ ).

\section{Does Size Matter?}

Starting from the late 1970s, a number of empirical evidences have shown that much of the variation in expected return is unrelated to the market beta. One of most prominent anomalies in asset pricing literature is the relation between return and firm size, first discovered by Banz (1981) and Reinganum (1981). For instance, Banz (1981), and Fama and French (1992) investigate a version of the following empirical model:

$$
r_{k}=a+b_{\beta} \beta_{k}^{M}+b_{\bar{y}} \bar{y}_{k}+\varepsilon_{k}, \quad k=1, \ldots, K
$$

where $a, b_{\beta}$, and $b_{\bar{y}}$ are regression coefficients assumed to be constant in their crosssectional analysis. They have found that size $\bar{y}_{k}$ is negatively correlated with return $r_{k}$, termed the size effect in the literature. Since the size effect was discovered first in the United States, it has been further confirmed in the United Kingdom and other markets. 
To explain the size effect, we note that the IaCAPM in (30) implies the following equation,

$$
r_{k}=\bar{r}_{M} \beta_{k}^{M}+\left[\left(c_{k}-1\right) \bar{r}_{M} \zeta_{M} / \lambda_{k} \sigma_{M M}\right] \bar{y}_{k}+\varepsilon_{k}, \quad k=1, \ldots, K .
$$

It is immediate from this equation that additional size variable $\bar{y}_{k}$ is required in order to explain the variation in return if we use market beta rather than information-adjusted beta to predict returns. Moreover, it can be demonstrated that the coefficient of $\bar{y}_{k}$ in the above equation is negative on average (the details are available upon request). This negative average size effect indicates that the IaCAPM's prediction does not contradict with the empirical findings of the size effect.

\section{Concluding Remarks}

This paper develops a new asset pricing model based on unconditional statistical analysis and illustrates that the assumption of complete agreement among all investors in a market is likely to be one of the major reasons for various anomalies discovered in the empirical tests of the classic CAPM. The reality of financial markets is that investors have diverse judgments and beliefs about the returns on risky assets and they frequently update their beliefs based on information they received. By removing the assumption of complete agreement, the Information-adjusted CAPM concludes that the unconditional expected return on a risky asset or a portfolio of assets is solely determined by the information-adjusted beta rather than the market beta. More specifically, it postulates that the unconditional expected return on a risky asset/portfolio is equal to the riskless interest rate plus the product of the informationadjusted beta and the unconditional expected excess return on the IaMP. Furthermore, the IaCAPM not only specifies the conditions under which the Sharpe-Lintner CAPM overstates or understates the expected return on a portfolio but also provides a formula to estimate the prediction error made by the Sharpe-Lintner CAPM. From a practical perspective, the IaCAPM introduces a new approach to analyze the empirical anomalies of the classic CAPM, including a flatter relation between average return and market 
beta than the Sharpe-Lintner CAPM predicts, a non-zero Jensen's alpha, the lack of explanatory power of market beta, and the size effect.

The empirical problems of the classic CAPM lead researchers to ask whether it is possible to empirically test the CAPM. The famous Roll's (1977) critique argues that the CAPM has never been and will probably never be tested because the theory of the CAPM does not clearly indicate which assets should be legitimately included in the market portfolio. So, when a proxy is used in a test, the test is actually testing the mean-variance efficiency of the proxy rather than the validity of the CAPM. In response to the Roll's critique, econometricians have devoted great efforts in searching for a better proxy of the market portfolio. Interestingly, various studies demonstrate that the test outcomes of the classic CAPM are not sensitive to the expansion of the market proxy beyond common stocks (Stambaugh (1982)). Even for a market portfolio including international assets, market beta still cannot explain the anomalies of the classic CAPM (Fama and French (1998)). While enlarging the "market" coverage to include more assets into the market portfolio is likely to make it closer to the "true" market portfolio, the IaCAPM suggests that the key for a successful test is not to extend the coverage of the market portfolio after it has a sufficiently large span. Rather, correct weights of the benchmark portfolio are more important. The market portfolio should be adjusted by the information index to reflect the uneven information distribution across assets. 


\section{REFERENCES}

Admati, Anat, 1985, A noisy rational expectations equilibrium for multi-asset securities markets, Econometrica 53, 629-658.

Banz, Rolf W., 1981, The relationship between return and market value of common stocks, Journal of Financial Economics 9, 3-18.

Biais, Bruno, 1993, Price formation and equilibrium liquidity in fragmented and centralized markets, Journal of Finance 48, 157-185.

Black, Fischer, 1972, Capital market equilibrium with restricted borrowing, Journal of Business 45, 444-455.

Black, Fischer, Michael C. Jensen, and Myron Scholes, 1972, The capital asset pricing model: Some empirical tests, in Michael C. Jensen, ed.: Studies in the Theory of Capital Markets (Praeger).

Campbell, John Y., 2000, Asset pricing at the millennium, Journal of Finance 55, $1515-1567$.

Cochrane, John H., 2005, Asset Pricing (Princeton University Press, Princeton and Oxford)

Easley, David, and Maureen O'Hara, 2004, Information and the cost of capital, Journal of Finance 59, 1553-1583.

Fama, Eugene E., and Kenneth R. French, 1992, The cross-section of expected stock returns, Journal of Finance 47, 427-465.

Fama, Eugene E., and Kenneth R. French, 1993, Common risk factors in the returns on stocks and bonds, Journal of Financial Economics 33, 3-56.

Fama, Eugene F., and Kenneth R. French, 1998, Value versus growth: The international evidence, Journal of Finance 53, 1975-999.

Fama, Eugene E., and Kenneth R. French, 2004, The capital asset pricing model: Theory and evidence, Journal of Economic Perspectives 18, 25-46.

Fitzmaurice, Garrett M., Nan M. Laird, and James H. Ware, 2004. Applied Longitudinal Analysis (John Wiley \&Sons, New York).

Grossman, Sanford, 1976, On the efficiency of competitive stock markets where trades have diverse information, Journal of Finance 31, 573-585. 
Grossman, Sanford, and Joseph E. Stiglitz, 1980, On the impossibility of informationally efficient markets, American Economic Review 70, 393-408.

Hellwig, Martin F., 1980, On the aggregation of information in competitive markets, Journal of Economic Theory 22, 477-498.

Hogg, Robert V., and Allen T. Craig, 1995. Introduction to Mathematical Statistics (Prentice Hall, Upper Saddle River, New Jersey).

Huang, Chi-Fu. and Robert H. Litzenberger, 1988. Foundations for Financial Economics (North Holland, New York).

Jagannathan, Ravi, and Zhenyu Wang, 1996, The conditional CAPM and the cross-section of expected returns, Journal of Finance 51, 3-53.

Lintner, John, 1965, The valuation of risk assets and the selection of risky investments in stock portfolios and capital budgets, Review of Economics and Statistics 47, 13-37.

Lintner, John, 1969, The aggregation of investor's diverse judgments and preferences in purely competitive securities markets, Journal of Financial and Quantitative Analysis $4,347-400$.

O'Hara, Maureen, 2003, President Address: Liquidity and Price Discovery, Journal of Finance 58, 1335-1353.

Reinganum, Marc R., 1981, Misspecification of capital asset pricing: Empirical anomalies based on earnings' yields and market values, Journal of Financial Economics 9, 19-46.

Roll, Richard, 1977, A critique of the asset pricing theory's tests: Part I: On past and potential testability of the theory, Journal of Financial Economics 4, 129-176.

Sharpe, William F., 1964, Capital asset prices: A theory of market equilibrium under conditions of risk, Journal of Finance 19, 425-442.

Stambaugh, Robert F., 1982, On the exclusion of assets from tests of the two parameter model, Journal of Financial Economics 10, 235-268.

Stoll, Hans R., 1978, The supply of dealer services in securities market, Journal of Finance $33,1133-1151$. 


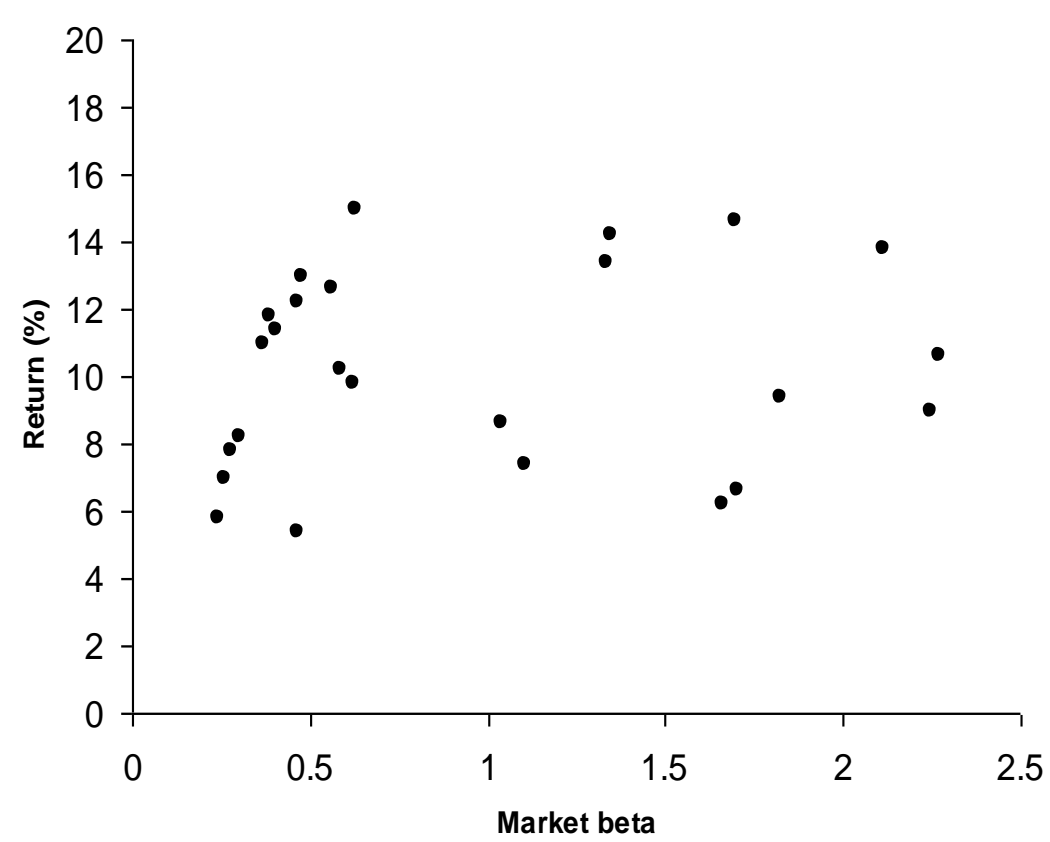

Figure 1a

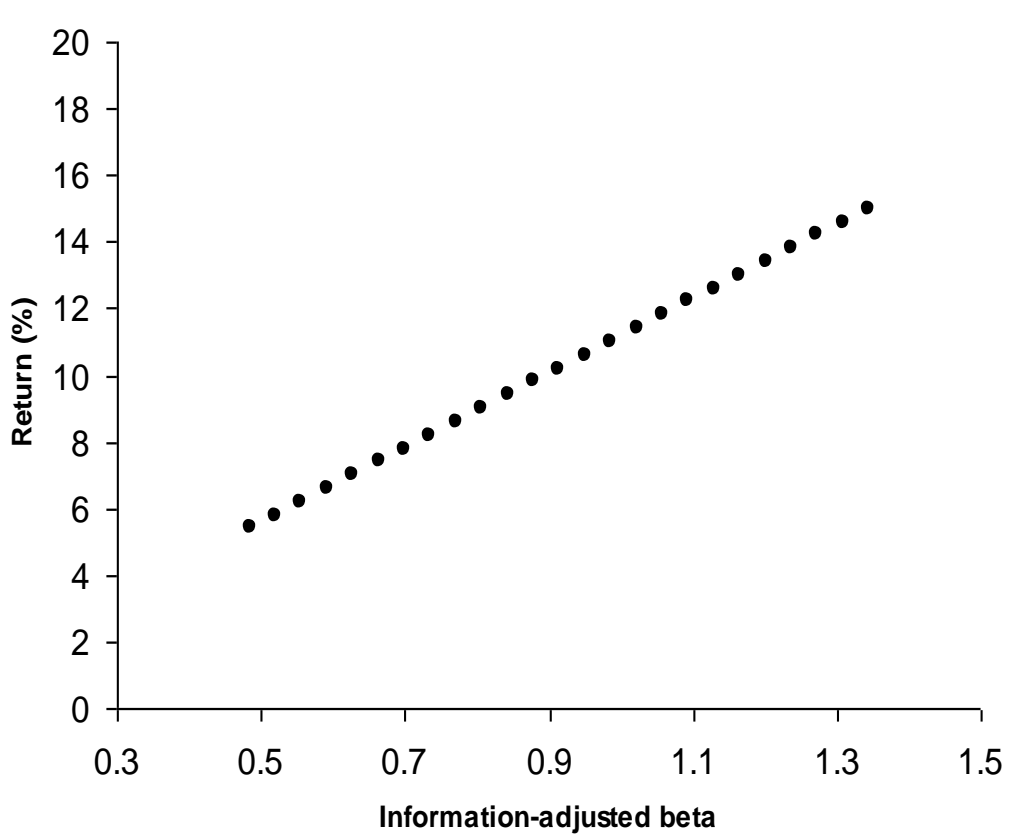

Figure 1b

Figure 1. Simulated return versus market beta or information-adjusted beta for a market of 25 stocks. Figure 1 a plots 25 points of market beta-returns $\left(\beta_{k}^{M}, \bar{r}_{k}\right)(k=1, \ldots, 25)$, which simulates conventional market observations and anomalies. Figure $1 \mathrm{~b}$ depicts 25 pairs of information-adjusted beta-returns $\left(\beta_{k}^{A}, \bar{r}_{k}\right)(k=1, \ldots, 25)$ for the same 25 stocks and they fall exactly on the straight-line of the IaCAPM. 


\begin{tabular}{|c|c|c|c|c|c|c|c|c|c|c|c|c|c|}
\hline$\omega_{k}$ & 0.106 & 0.110 & 0.114 & 0.132 & 0.146 & 0.185 & 0.191 & 0.235 & 0.244 & 0.285 & 0.299 & 0.328 & 0.445 \\
\hline $\bar{r}_{k}-\bar{r}_{M} \beta_{k}^{M}$ & -12.072 & -12.159 & -15.753 & -14.440 & -10.678 & -9.468 & -4.740 & -2.838 & -4.095 & -1.283 & -0.656 & 0.254 & 2.922 \\
\hline$\omega_{k}$ & 0.495 & 0.635 & 0.676 & 0.685 & 0.742 & 0.761 & 0.771 & 0.775 & 0.798 & 0.804 & 0.852 & 0.872 & \\
\hline $\bar{r}_{k}-\bar{r}_{M} \beta_{k}^{M}$ & 3.764 & 6.401 & 8.070 & 3.154 & 7.059 & 4.126 & 4.878 & 7.761 & 4.744 & 6.970 & 6.964 & 7.574 & \\
\hline$\left|\omega_{k}-\omega^{*}\right|$ & 0.014 & 0.015 & 0.027 & 0.068 & 0.078 & 0.122 & 0.127 & 0.133 & 0.166 & 0.180 & 0.183 & 0.199 & 0.203 \\
\hline$\left|\bar{r}_{k}-\bar{r}_{M} \beta_{k}^{M}\right| /\left|\bar{r}_{M} \beta_{k}^{M}\right|$ & 0.044 & 0.049 & 0.087 & 0.219 & 0.248 & 0.390 & 0.407 & 0.425 & 0.532 & 0.577 & 0.585 & 0.636 & 0.648 \\
\hline$\left|\omega_{k}-\omega^{*}\right|$ & 0.206 & 0.323 & 0.364 & 0.372 & 0.429 & 0.448 & 0.459 & 0.463 & 0.485 & 0.492 & 0.539 & 0.560 & \\
\hline$\left|\bar{r}_{k}-\bar{r}_{M} \beta_{k}^{M}\right| /\left|\bar{r}_{M} \beta_{k}^{M}\right|$ & 0.661 & 1.033 & 1.165 & 1.192 & 1.373 & 1.435 & 1.468 & 1.481 & 1.553 & 1.573 & 1.726 & 1.792 & \\
\hline
\end{tabular}

Table 1. The absolute and relative prediction errors of the Sharpe-Lintner CAPM. The upper panel shows that when $\omega_{k}<\omega^{*}=0.31\left(\omega_{k}>\omega^{*}=0.31\right)$ the prediction errors of the Sharp-Lintner CAPM are negative (positive) so that the Sharpe-Lintner CAPM overstates (understates) unconditional expected returns. The lower panel demonstrates the conclusion of Proposition 4 that the relative error made by the Sharpe-Lintner CAPM increases monotonically and is proportionate to $\left|\omega_{k}-\omega^{*}\right|$. 


\section{Appendix A. Proofs of Propositions and Corollaries}

Proof of Proposition 1: Substituting equation (15) into (17), we obtain

$$
\kappa_{k} \lambda_{k}^{I} \overline{\ln }_{k}^{I}+\left(1-\kappa_{k}\right) \lambda_{k}^{U} \overline{\ln v_{k}^{U}}-\delta y_{k}=\left[\kappa_{k} \lambda_{k}^{I}+\left(1-\kappa_{k}\right) \lambda_{k}^{U}\right] \ln p_{k} .
$$

Since $\lambda_{k}^{M}=\kappa_{k} \lambda_{k}^{I}+\left(1-\kappa_{k}\right) \lambda_{k}^{U}$ as defined in (21), substituting (6)-(7) and (11)-(12) into (A1) and then using (9) to eliminate $\theta_{k}$ yield

$$
\begin{aligned}
\ln p_{k}= & \left(\lambda_{k}^{M}\right)^{-1}\left\{\rho_{k} \overline{\ln v_{k}}+\left[\kappa_{k} \gamma_{k} \alpha_{k} I_{k}+\left(1-\kappa_{k}\right) \lambda_{\theta k}\right] g_{k}+\gamma_{k}\left(1-\alpha_{k}\right) I_{k} h_{k}\right. \\
& \left.-\left[\delta+\left(1-\kappa_{k}\right) \lambda_{\theta k} B_{y} / B_{g}\right] y_{k}+\left(1-\kappa_{k}\right) \lambda_{\theta k}\left(B_{y} / B_{g}\right) \bar{y}_{k}\right\} .
\end{aligned}
$$

Comparing the coefficients of $g_{k}$ and $y_{k}$ with their counterparts in (9), we obtain

$$
\frac{B_{y}}{B_{g}}=-\frac{\delta+\left(1-\kappa_{k}\right) \lambda_{\theta k} B_{y} / B_{g}}{\kappa_{k} \gamma_{k} \alpha_{k} I_{k}+\left(1-\kappa_{k}\right) \lambda_{\theta k}},
$$

which in turn requires $B_{y} / B_{g}=\delta / \kappa_{k} \gamma_{k} \alpha_{k} I_{k}$. Inserting it into (A2) yields the coefficients:

$$
\begin{array}{ll}
B_{v}=\rho_{k} / \lambda_{k}^{M}, & B_{h}=\gamma_{k}\left(1-\alpha_{k}\right) I_{k} / \lambda_{k}^{M} \\
B_{g}=\left[\kappa_{k} \gamma_{k} \alpha_{k} I_{k}+\left(1-\kappa_{k}\right) \lambda_{\theta k}\right] / \lambda_{k}^{M}, & B_{y}=\delta\left[1+\left(1-\kappa_{k}\right) \lambda_{\theta k} / \kappa_{k} \gamma_{k} \alpha_{k} I_{k}\right] / \lambda_{k}^{M}, \\
B_{\bar{y}}=\delta\left(1-\kappa_{k}\right) \lambda_{\theta k} / \kappa_{k} \gamma_{k} \alpha_{k} I_{k} \lambda_{k}^{M}, & \lambda_{\theta k}=\left[\delta^{2}\left(\kappa_{k} \gamma_{k} \alpha_{k} I_{k}\right)^{-2} \eta_{k}^{-1}+\left(\gamma_{k} \alpha_{k} I_{k}\right)^{-1}\right]^{-1} .
\end{array}
$$

Proof of Proposition 2: Note that

$$
E\left[\ln \left(v_{k} / p_{k}\right)\right]=\overline{\ln v_{k}}-\left[B_{v} \overline{\ln v_{k}}+B_{g} E\left(g_{k}\right)+B_{h} E\left(h_{k}\right)-\left(B_{y}-B_{\bar{y}}\right) \bar{y}_{k}\right] .
$$

Recalling $E\left(g_{k}\right)=\overline{\ln v_{k}}, E\left(h_{k}\right)=\overline{\ln v_{k}}$ and $B_{v}+B_{g}+B_{h}=1$, substituting the values of $B_{y}$ and $B_{\bar{y}}$ in Proposition 1 into (A3), we obtain (22). To compute $\operatorname{var}\left(r_{k}\right)$ we first note the following identity:

$$
\operatorname{var}\left(r_{k}\right)=E\left[\operatorname{var}\left(r_{k} \mid v_{k}\right)\right]+\operatorname{var}\left[E\left(r_{k} \mid v_{k}\right)\right] .
$$

Since $\operatorname{var}\left(g_{k} \mid v_{k}\right)=\left(\gamma_{k} \alpha_{k} I_{k}\right)^{-1}, \operatorname{var}\left(h_{k} \mid v_{k}\right)=\left[\gamma_{k}\left(1-\alpha_{k}\right) I_{k}\right]^{-1}$ and $\operatorname{var}\left(y_{k}\right)=\eta_{k}^{-1}$, we have

$$
\begin{aligned}
\operatorname{var}\left(r_{k} \mid v_{k}\right) & =\operatorname{var}\left(B_{g} g_{k}+B_{h} h_{k}-B_{y} y_{k} \mid v_{k}\right) \\
& =B_{g}^{2}\left(\gamma_{k} \alpha_{k} I_{k}\right)^{-1}+B_{h}^{2}\left[\gamma_{k}\left(1-\alpha_{k}\right) I_{k}\right]^{-1}+B_{y}^{2} \eta_{k}^{-1} .
\end{aligned}
$$

Moreover, $E\left[\operatorname{var}\left(r_{k} \mid v_{k}\right)\right]=\operatorname{var}\left(r_{k} \mid v_{k}\right)$ since $\operatorname{var}\left(r_{k} \mid v_{k}\right)$ is a constant. On the other hand, (18) shows 


$$
E\left(r_{k} \mid v_{k}\right)=\ln v_{k}-\left[B_{v} \overline{\ln v_{k}}+B_{g} E\left(g_{k} \mid v_{k}\right)+B_{h} E\left(h_{k} \mid v_{k}\right)+\left(B_{y}-B_{\bar{y}}\right) \bar{y}_{k}\right] .
$$

Inserting $E\left(g_{k} \mid v_{k}\right)=\ln v_{k}$ and $E\left(h_{k} \mid v_{k}\right)=\ln v_{k}$ into it, we obtain $E\left(r_{k} \mid v_{k}\right)=B_{v} \ln v_{k}+$ constant, which implies $\operatorname{var}\left[E\left(r_{k} \mid v_{k}\right)\right]=B_{v}^{2} \rho_{k}^{-1}$. Substituting the last equation and (A5) into (A4) yields (23).

Proof of Proposition 3: By definition,

$$
\begin{aligned}
& \sigma_{k A}=E\left[\left(r_{k}-\bar{r}_{k}\right)\left(\sum_{j=1}^{K} t_{j}^{A}\left(r_{j}-\bar{r}_{j}\right)\right)\right]=t_{k}^{A} / \lambda_{k}, \\
& \sigma_{A A}=E\left[\left(\sum_{k=1}^{K} t_{k}^{A}\left(r_{k}-\bar{r}_{k}\right)\right)^{2}\right]=\sum_{k=1}^{K}\left(t_{k}^{A}\right)^{2} / \lambda_{k} .
\end{aligned}
$$

From (22), we have $\bar{r}_{k} / \delta=\bar{y}_{k} / \lambda_{k}^{M}$. Substituting $t_{k}^{A}$ into it and then using $\sigma_{k A}$, we obtain

$$
\bar{r}_{k} / \delta=t_{k}^{A} / \lambda_{k} \zeta_{A}=\sigma_{k A} / \zeta_{A} .
$$

Multiplying both sides of it by $t_{k}^{A}$ and then summing over all $k$ yield

$$
\bar{r}_{A} / \delta=\left[\sum_{k=1}^{K}\left(t_{k}^{A}\right)^{2} / \lambda_{k}\right] / \zeta_{A}=\sigma_{A A} / \zeta_{A} .
$$

The combination of the last two equations yields (24). Multiplying both sides of (24) by $t_{k}$ and then summing over all $k$ yield (25).

Proof of Proposition 4: From (24), we have $\bar{r}_{k}=c \omega_{k} \bar{r}_{M} \beta_{k}^{M}$ for all $k$. Since $\omega_{k} \in(0,1)$ is not constant across $k$, so is $c \omega_{k}$. Suppose $c \omega_{k}<1$ for all $k$. Then, $\bar{r}_{k}<\bar{r}_{M} \beta_{k}^{M}$ for all $k$. Multiplying both sides by $t_{k}^{M}>0$ and summing over all $k$ yield $\bar{r}_{M}<\bar{r}_{M} \sum_{k=1}^{n} t_{k}^{M} \sigma_{k M} / \sigma_{M M}$ $=\bar{r}_{M}$. This contradiction implies that the assumption of $c \omega_{k}<1$ for all $k$ is false. By the same logic, $c \omega_{k}>1$ for all $k$ is not true either. Define $\omega^{*} \equiv c^{-1}$. Obviously, $\omega^{*} \in\left(\min _{k}\left\{\omega_{k}\right\}, \max _{k}\left\{\omega_{k}\right\}\right) \subseteq(0,1)$ is unique. On the other hand, manipulating (25) yields $\bar{r}_{P}=c \omega_{P} \bar{r}_{M} \beta_{P}^{M}$. Therefore, $\left|\bar{r}_{P}-\bar{r}_{M} \beta_{P}^{M}\right| /\left|\bar{r}_{M} \beta_{P}^{M}\right|=c\left|\omega_{P}-\omega^{*}\right|$.

Apparently, $c \omega_{P}<1$ if $\omega_{P} \in\left(0, \omega^{*}\right)$. Hence, $\bar{r}_{P}<\bar{r}_{M} \beta_{P}^{M}$ if $\omega_{P} \in\left(0, \omega^{*}\right)$ and $\beta_{P}^{M}>0$. Similarly, $\bar{r}_{P}>\bar{r}_{M} \beta_{P}^{M}$ if $\omega_{P} \in\left(\omega^{*}, 1\right)$ and $\beta_{P}^{M}>0$.

Finally, $t_{k}^{M}=t_{k}^{A}$ for all $k$ when $\omega_{k}$ is constant across securities. Then, the IaMP and IaCAPM collapse to the market portfolio and Sharpe-Lintner CAPM, respectively. 
Proof of Lemma 1: Claim (iii) is obvious so we first consider (i)-(ii). For simplicity, we suppress subscript $k$, then

$$
\begin{aligned}
\omega & =\frac{\lambda}{\lambda^{M}}=\frac{\rho+(1-\alpha) \gamma I+\alpha \kappa \gamma I+(1-\kappa) \lambda_{\theta}}{\left.\rho+(1-\alpha) \gamma I+\left[1+(1-\kappa) \lambda_{\theta} / \kappa \gamma \alpha I\right)\right]^{2}\left(\kappa^{2} \gamma \alpha I+\delta^{2} / \eta\right)} \\
& =\frac{\rho / \gamma I+1-\alpha+\alpha \kappa+(1-\kappa) \lambda_{\theta} / \gamma I}{\rho / \gamma I+1-\alpha+\left[1+(1-\kappa) \lambda_{\theta} / \kappa \gamma \alpha I\right]^{2}\left(\kappa^{2} \alpha+\delta^{2} / \eta \gamma I\right)},
\end{aligned}
$$

where $\lambda_{\theta}=\alpha(\gamma I)^{2} /\left(\gamma I+\delta^{2} / \eta \alpha \kappa^{2}\right)$. There are three cases under $\Psi \rightarrow+\infty$. First, consider the case that $\gamma I$ is finite and $\Psi \rightarrow+\infty$. This implies $\rho \rightarrow+\infty$, and clearly we have $\lim _{\rho \rightarrow+\infty} \omega=1$. Second, $\rho$ is finite and $\Psi \rightarrow+\infty$. This implies $\gamma I \rightarrow+\infty$. Noting $\lim _{\gamma I \rightarrow+\infty} \lambda_{\theta} / \gamma I=\alpha$, we obtain

$$
\lim _{\gamma I \rightarrow+\infty} \omega=\frac{\lim _{\gamma I \rightarrow+\infty} \rho / \gamma I+1-\alpha+\alpha \kappa+(1-\kappa) \alpha}{\lim _{\gamma I \rightarrow+\infty} \rho / \gamma I+1-\alpha+\alpha}=1 .
$$

Finally, we consider the case that both $\rho \rightarrow+\infty$ and $\gamma I \rightarrow+\infty$. Since $\lim _{\substack{\gamma I \rightarrow+\infty \\ \rho \rightarrow+\infty}} \rho / \gamma I$ exists, we obtain $\lim _{\psi \rightarrow+\infty} \omega=\frac{\lim _{\gamma \rightarrow \rightarrow+\infty, \rho \rightarrow+\infty} \rho / \gamma I+1}{\lim _{\gamma \rightarrow+\infty, \rho \rightarrow+\infty} \rho / \gamma I+1}=1$.

In addition, since $\lambda^{M}=\rho+(1-\alpha) \gamma I+\alpha \kappa \gamma I+(1-\kappa) \lambda_{\theta}$, it is easy to verify that $\lim _{\psi \rightarrow+\infty} \lambda^{M}=+\infty$. Hence, $\lim _{\psi \rightarrow+\infty} \lambda=\lim _{\psi \rightarrow+\infty} \lambda^{M} \omega=+\infty$.

When $\Psi \rightarrow 0$, it implies both $\rho \rightarrow 0$ and $\gamma I \rightarrow 0$. Noting $\lim _{\gamma I \rightarrow 0} \lambda_{\theta}=0$ and $\lim _{\gamma \rightarrow 0} \lambda_{\theta} / \gamma I=0$, it is clear that $\lim _{\psi \rightarrow 0} \omega=0$. Once again, we can easily obtain $\lim _{\Psi \rightarrow 0} \lambda=\lim _{\psi \rightarrow 0} \lambda^{M} \omega=0$.

Now we show (iv)-(vi). It has been shown that $\lambda_{k}^{M} \rightarrow+\infty$ and $\lambda_{k} \rightarrow+\infty$ with the same order when $\Psi_{k} \rightarrow+\infty$. So, from the definition of $\omega_{P}$, it is immediate that $\omega_{P} \rightarrow 1$ when $\Psi_{P} \rightarrow+\infty$ because $\Psi_{P} \rightarrow+\infty$ implies $\Psi_{k} \rightarrow+\infty$ for all $k$. Next, $\Psi_{P} \rightarrow 0$ implies that there exists at least one $k$ such that $\Psi_{k} \rightarrow 0$. Since $\lambda_{k}^{M} \rightarrow 0$ and $\lambda_{k} \rightarrow 0$ but $\omega_{k}=\lambda_{k} / \lambda_{k}^{M} \rightarrow 0$ when $\Psi_{k} \rightarrow 0$, we have $\omega_{P} \rightarrow \omega_{k} \rightarrow 0$. Finally, recalling $0<\lambda_{k}<\lambda_{k}^{M}$ for all $k,(v i)$ is obvious from the definition of $\omega_{P}$. 
Proof of Proposition 5: In empirical tests of the classic CAPM along the line of Fama and French (1992, 1993), all portfolios have non-negative weights and therefore their information indexes satisfy $\omega_{P} \in(0,1)$ according to Lemma 1. Furthermore, Lemma 1 implies that there exists a small and positive constant $\Psi_{L}$ such that $\omega_{P}$ falls into interval $\left(0, \omega^{*}\right)$ if $\Psi_{P}<\Psi_{L}$. For all these portfolios, Proposition 4 shows that the Sharpe-Lintner CAPM overstates their expected returns. The proof for part (ii) is similar.

Proof of Corollary 1: Define $\beta_{U} \equiv a_{0} a_{U} h_{\min } \Psi_{L}^{-1}$, where $h_{\min }=\min _{k}\left\{\Psi_{k} / \lambda_{k}\right\}$, $a_{0} \equiv\left(\sigma_{M M} \sum_{k=1}^{K} \bar{y}_{k}\right)^{-1}$ and $a_{\min } \equiv \min _{t_{k} \geq 0}\left\{\sum_{k=1}^{K} t_{k} \bar{y}_{k} \mid \sum_{k=1}^{K} t_{k}=1\right\}$. For a portfolio in set $P_{L}$, there is $\sum_{k=1}^{K} \pi_{k} \Psi_{k}^{-1}>\Psi_{L}^{-1}$. Then, its market beta satisfies $\beta_{P}^{M}=a_{0} \sum_{k=1}^{K} t_{k} \bar{y}_{k} / \lambda_{k}>$ $a_{0} h_{\min } \sum_{k=1}^{K} t_{k} \bar{y}_{k} / \Psi_{k} \geq a_{0} a_{\min } h_{\min } \sum_{k=1}^{K} \pi_{k} / \Psi_{k}>\beta_{U}$. The proof for portfolios in $P_{U}$ is similar but $\beta_{L}$ should be defined as $\beta_{L} \equiv a_{0} a_{\max } h_{\max } \Psi_{U}^{-1}$, where $h_{\max }=\max _{k}\left\{\Psi_{k} / \lambda_{k}\right\}$ and $a_{\max } \equiv \max _{t_{k} \geq 0}\left\{\sum_{k=1}^{K} t_{k} \bar{y}_{k} \mid \sum_{k=1}^{K} t_{k}=1\right\}$.

Proof of Corollary 2: The proof is obvious so that it is omitted. 